\title{
Cue-motivated reward seeking is negatively regulated by expected reward magnitude in Pavlovian-instrumental transfer
}

\section{Andrew T. Marshall ${ }^{1}$ and Sean B. Ostlund ${ }^{2}$}

1. Children's Hospital Los Angeles, and the Department of Pediatrics, University of Southern California, Los Angeles, CA, USA

2. Department of Anesthesiology and Perioperative Care, Department of Neurobiology and Behavior, Irvine Center for Addiction Neuroscience, Center for the Neurobiology of Learning and Memory, University of California, Irvine School of Medicine, Irvine, CA, USA

Correspondence Dr Sean Ostlund, Department of Anesthesiology \& Perioperative Care, University of California, Irvine School of Medicine, Irvine, CA 92697, USA. E-mail:

sostlund@uci.edu 


\section{Abstract}

The Pavlovian-instrumental transfer (PIT) paradigm is widely used to assay the motivational influence of reward-paired cues, which is reflected by their ability to stimulate instrumental reward-seeking behavior. Leading models of incentive learning assume that motivational value is assigned to cues based on the total amount of reward they signal (i.e., their state value). Based on recent findings, we lay out the alternative hypothesis that cue-elicited reward predictions may actually suppress the motivation to seek out new rewards through instrumental behavior in order to facilitate efficient retrieval of a reward that is already expected, before it is lost or stolen. According to this view, cue-motivated reward seeking should be inversely related to the magnitude of an expected reward, since there is more to lose by failing to secure a large reward than a small reward. We investigated the influence of expected reward magnitude on PIT expression. Hungry rats were initially trained to lever press for food pellets before undergoing Pavlovian conditioning, in which two distinct auditory cues signaled food pellet delivery at cue offset. Reward magnitude was varied across cues and groups. While all groups had at least one cue that signaled three food pellets, the alternate cue signaled either one (Group 1/3), three (Group 3/3), or nine food pellets (Group 3/9). PIT testing revealed that the motivational influence of reward-predictive cues on lever pressing varied inversely with expected reward magnitude, with the 1-pellet cue augmenting performance and the 3- and 9-pellet cues suppressing performance, particularly near the expected time of reward delivery. This pattern was mirrored by opposing changes in the food-port entry behavior, which varied positively with expected reward magnitude. We discuss how these findings may relate to cognitive control over cue-motivated behavior. 
Reward-paired cues acquire motivational properties which allow them to facilitate the expression of instrumental reward-seeking behavior, a phenomenon referred to as Pavlovian-instrumental transfer (PIT)(Holmes et al., 2010; Cartoni et al., 2016; Corbit and Balleine, 2016). The PIT paradigm is widely used to study the mechanisms of Pavlovian incentive motivation and how they contribute to pathological reward seeking in addiction and related disorders in both humans (Garbusow et al., 2016; Vogel et al., 2018; Genauck et al., 2020; Sebold et al., 2021) and animal models (Wyvell and Berridge, 2001; Corbit and Janak, 2007; Saddoris et al., 2011; LeBlanc et al., 2012; Shiflett, 2012; LeBlanc et al., 2013; Shiflett et al., 2013; LeBlanc et al., 2014; Ostlund et al., 2014; Corbit and Janak, 2016; Marshall and Ostlund, 2018). However, despite decades of research, the specific factors that regulate the expression of PIT are not well understood.

Leading computational models of incentive learning (Dayan and Balleine, 2002;

McClure et al., 2003; Zhang et al., 2009) assume that the motivational value assigned to cue is directly related to the total amount of delay-discounted reward expected (i.e., its state value), and should therefore be sensitive to manipulations of Pavlovian conditioning parameters such as reward probability, timing, and magnitude. Specifically, cues that reliably signal the immediate delivery of a large reward should acquire strong motivational properties, whereas cues signaling that reward will be small, delayed, or unlikely to occur at all, should acquire weak motivational properties. 
However, there is growing evidence that the motivational impact of reward-paired cues may instead be inversely related to the amount of reward they predict. For instance, cues signaling that there is a high probability of imminent reward do not invigorate and may even suppress instrumental performance (Azrin and Hake, 1969; Vandyne, 1971; Lovibond, 1981; Crombag et al., 2008), with this behavior being replaced by increased levels of focal-search behavior directed at the food-port (Marshall et al., 2020). In contrast, cues signaling a low probability of imminent reward are more likely to invigorate instrumental reward seeking and less likely to elicit food-port entries (Marshall et al., 2020). Cues signaling a long delay until reward delivery produce a more complex pattern of behavior, eliciting a gradual transition from pressing the lever to checking the food-port as the expected time of reward delivery draws near (Marshall and Ostlund, 2018).

To our knowledge the effects of expected reward magnitude on PIT performance has not been directly investigated. However, it is well-established that post-training reward devaluation has little to no impact on the expression of cue-motivated lever pressing (Colwill and Rescorla, 1988; Rescorla, 1994; Holland, 2004), suggesting that the motivational influence of a cue is not inherently connected to the expected value of the reward that it has come to signal.

Such findings suggest that reward-paired cues acquire independent motivational and predictive properties that can lead to conflicting response strategies, with the former promoting the pursuit of new rewards through instrumental action and the latter 
facilitating the retrieval of a reward that is expected soon. This would help ensure foraging efficiency, as it is more adaptive to engage in exploratory reward-seeking behavior when rewards are scarce or difficult to obtain. In contrast, when there is reason to expect imminent reward, it is best to avoid unnecessary reward seeking and focus on retrieving the expected reward before it is pilfered or otherwise lost.

Following this logic, we have recently argued that the expression of PIT is regulated by cognitive control in order to suppress instrumental reward seeking and facilitate reward retrieval when a cue signals a strong reward expectancy (Marshall and Ostlund, 2018; Marshall et al., 2020). One important prediction of this view is that the magnitude of the expected reward effectively sets the stakes for the decision to seek or retrieve, since it is more important to secure a large reward than a small reward. We tested this hypothesis in the current PIT study by performing a parametric assessment of expected reward magnitude on expression of cue-evoked instrumental lever pressing and concomitant food-port entry behavior.

\section{Methods}

\section{Animals and Apparatus}

Twenty-six experimentally naïve adult male Long Evans rats (Envigo) were used in this experiment. They arrived at the facility (University of California, Irvine; Irvine, CA, USA) at approximately 10 weeks of age. They were pair-housed in a colony room set to a standard 12:12 hr light:dark schedule. The rats were tested during the light phase. Water was always provided ad libitum in the home cages. Rats were fed between 10-14 
$\mathrm{g}$ of standard lab chow per day during the experiment to maintain them at $\sim 85 \%$ of their estimated free-feeding bodyweight. Husbandry and experimental procedures were approved by the UC Irvine Institutional Animal Care and Use Committee (IACUC) and were in accordance with the National Research Council Guide for the Care and Use of Laboratory Animals.

The experiment was conducted in 14 operant chambers (Med-Associates; St. Albans, VT), each housed within sound-attenuating, ventilated boxes. Each chamber was equipped with a stainless-steel grid floor; two stainless steel walls (front and back); and a transparent polycarbonate side-wall, ceiling, and door. Pellet dispensers, mounted on the outside of the operant chamber, were equipped to deliver 45-mg food pellets (BioServ) to a recessed food cup centered on the lower section of the front wall. Head entries into the food port were transduced by an infrared photobeam. A retractable lever was located to the left of the food cup, on the front wall. The chamber was also equipped with a house light centered at the top of the back wall. Auditory stimuli were presented to animals via a speaker located on the back wall. Experimental events were controlled and recorded with 10-ms resolution by the software program MED-PC IV (Tatham and Zurn, 1989).

\section{Procedure}

Magazine training. All sessions of all phases began with the onset of the houselight. In each of two 30-minute sessions of magazine training, food pellets were delivered on a random-time (RT) 60-s schedule. 
Instrumental training. During initial instrumental (lever-press) training, rats were continuously reinforced with food pellet delivery for pressing the left lever (fixed-ratio, FR-1), earning a maximum of 30 pellets per session. These FR-1 sessions lasted no more than 30 min. Seven rats required an extra session of FR-1 training, which lasted until these rats earned 30 pellets. During subsequent training sessions, lever pressing was reinforced according to a random-interval $(\mathrm{RI})$ schedule, such that the lever remained available but was inactive for an average of $t$ seconds after each reward delivery, where individual $t$ values were selected randomly from an exponential distribution. The RI schedule was changed over training days with 1 day of $\mathrm{RI}-5$ ( $t=5$ sec), 1 day of $\mathrm{RI}-15$ ( $t=15 \mathrm{sec}), 2$ days of $\mathrm{RI}-30$ ( $t=30 \mathrm{sec})$, and 10 days of $\mathrm{RI}-45(t=$ $45 \mathrm{sec}$ ) training. Each $\mathrm{RI}$ session lasted 30 minutes.

Pavlovian training. Pavlovian training involved exposure to two 30 -s conditioned stimuli (CS; 3-kHz tone and 10-Hz clicker) paired with reward (food pellets). Rats were assigned to one of three groups with different CS-reward magnitudes. For Group 1/3 ( $n$ = 9), one CS terminated with 1 pellet; the other CS, 3 pellets. For Group 3/3 $(n=8)$, both CSs terminated with 3 pellets. For Group 3/9 $(n=9)$, one CS terminated with 3 pellets and the other with 9 pellets.

In each 20-min session, a 60-s interval preceded onset of the first CS. There was a 120s inter-stimulus interval (ISI) between consecutive CS presentations (i.e., between previous CS offset and subsequent CS onset), and a 60-s interval following the final CS 
presentation prior to the end of the session. Pavlovian training lasted for 25 sessions, each involving 4 pseudorandomly-alternating presentations of each CS (8 total trials per session).

Pavlovian-to-instrumental transfer (PIT). Following Pavlovian training, rats received two sessions of instrumental retraining (identical to earlier sessions with RI45 schedule) followed by one 30-min session of instrumental extinction, in which the lever was continuously available but was inactive. Rats then received a PIT test session $(30.25$ minutes in duration), during which the lever was once again continuously available but inactive. During the test, rats received 4 noncontingent presentations of each 30 -s CS in pseudorandom order (ABBABAAB). The ISI was $150 \mathrm{~s}$, and a 5-min interval preceded onset of the first CS. No food pellets were delivered at test.

\section{Data Analysis}

All summary measures were obtained from the raw data using MATLAB (The MathWorks; Natick, MA, USA), and analyzed with mixed-effects regression models (Pinheiro and Bates, 2000), a powerful analytical framework that is both well established and highly recommended for behavioral research (Boisgontier and Cheval, 2016). Mixed-effects models are comparable to repeated-measures regression analyses, and allow for parameter estimation per manipulation condition (fixed effects) and the individual (random effects) (Pinheiro and Bates, 2000; Hoffman and Rovine, 2007; Bolker et al., 2009; Schielzeth et al., 2013). Mixed-effects regression models (1) effectively handle missing data and (2) permit the inclusion of categorical and 
continuous predictors in the same analysis, thus allowing detection of group-level changes across ordered data samples (i.e., continuous time points) while also accounting for corresponding individual differences. All relevant fixed-effects factors were included in each model. Categorical predictors were effects-coded (i.e., codes sum to 0), and continuous predictors were mean-centered. For analyses of Pavlovian training and PIT, the fixed-effects structure included main effects of group and reward magnitude, and the random-effects structure included a by-rat random intercept. Instrumental training analyses incorporated generalized linear mixed-effects models (family: gamma, link: log) with predictors of group and time since the previous reward delivery. The random-effects structure include a random by-rat slope of time since reward delivery and a random intercept of rat, restricted to be uncorrelated. The alpha level for all tests was .05. Sample sizes were not predetermined based on statistical analysis but are similar to those reported in previous publications (Marshall and Ostlund, 2018; Halbout et al., 2019; Marshall et al., 2020). Main effects and interactions are reported as the results of ANOVA F-tests (i.e., whether the coefficients for each fixed effect were significantly different from 0 ).

Our primary dependent measures were lever pressing and food-port entry behavior. We quantified cue-induced changes in behavior by subtracting the mean response rate during pre-CS periods (30 sec each) from the mean response rate during CS periods (30 sec each). Pre-CS (baseline) data were averaged across all CS trials (within subject). Food-port entry behavior can fall into two categories: spontaneous entries and entries that are performed as part of an instrumental press-entry action sequence 
(Marshall and Ostlund, 2018; Halbout et al., 2019; Marshall et al., 2020), the latter indicated by an increased likelihood of food-port entry shortly after execution of the lever-press response. To focus our analysis on cue-elicited (and not instrumental) reward-retrieval behavior, we excluded all entries that occurred within a $2.5 \mathrm{sec}$ postlever press period from our analysis, as in our previous studies (Marshall and Ostlund, 2018; Halbout et al., 2019; Marshall et al., 2020). The final five sessions of Pavlovian training were used to assess conditioned food-port entry behavior during CS+ and CStrials relative to pre-CS baseline periods. Analyses of instrumental training included the final three sessions of training.

\section{Results}

\section{Instrumental and Pavlovian Training}

Rats were first trained to lever press for food reward. While the rats had not yet been assigned to groups for Pavlovian training (i.e., reward magnitude following CS termination), Figure $1 \mathrm{~A}$ shows the groups' mean lever-press rates on an RI-45 s schedule of reinforcement as a function of time since previous reward delivery. Per a generalized linear mixed-effects model (distribution $=$ gamma, link $=\log$ ) on response rates during the 15-45 s time window, there was neither a main effect of group, $F(1$, $800)=0.21, p=.811$, nor a Group $\times$ Time interaction, $F(2,800)=0.57, p=.566$. 

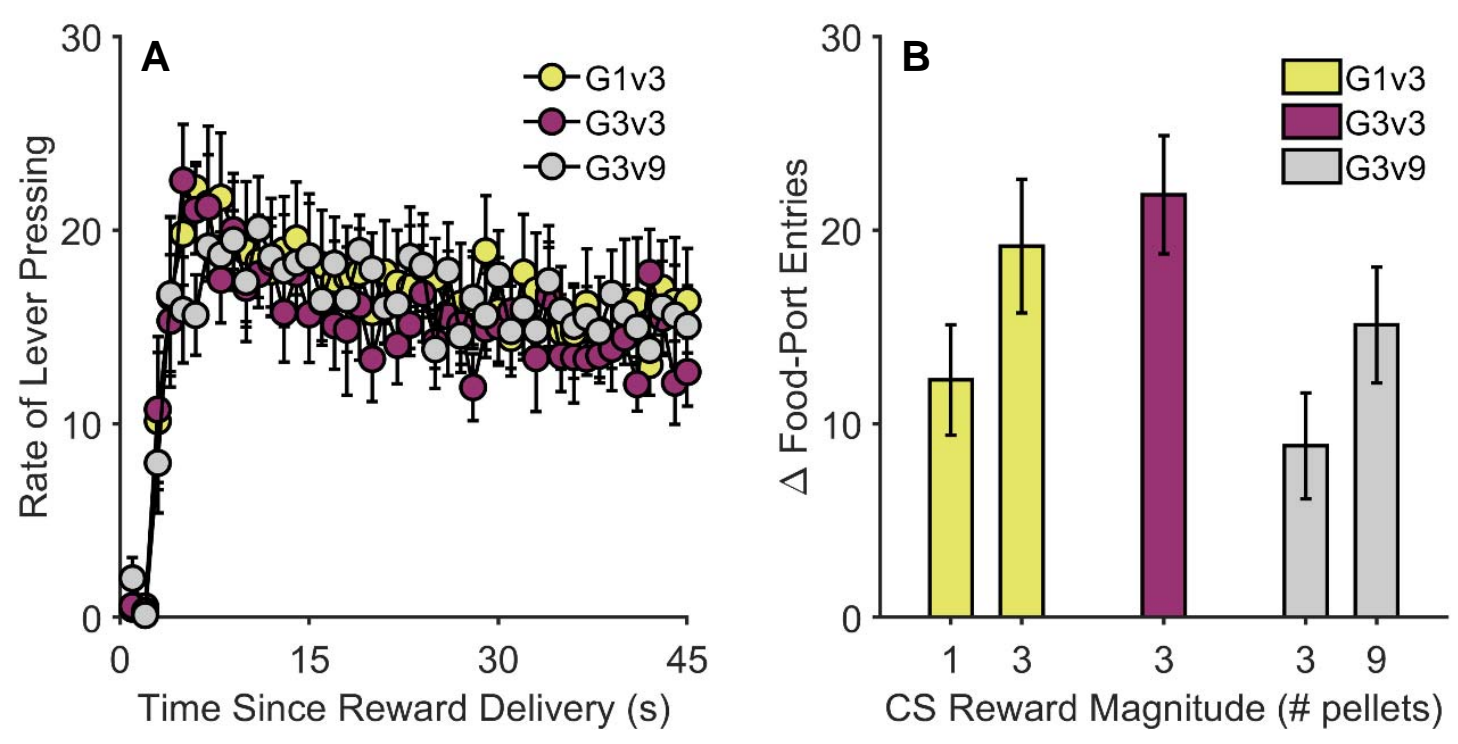

Figure 1. Instrumental and Pavlovian training. (A) Lever-press rates on a random-interval (RI) 45-s schedule of reinforcement as a function of time (in seconds) since previous reward delivery. (B) CS-induced increase in food-port entries relative to the pre-CS baseline. $\mathrm{CS}=$ conditioned stimulus. G1v3 = Group 1/3. G3v3 = Group 3/3. G3v9 = Group 3/9.

Following instrumental training, rats were trained to associate two 30-s CS cues with food reward, delivered upon termination of the cues. Across the final 5 sessions of training, for Groups $1 / 3$ and 3/9, rats showed increased food-port entry behavior (relative to the pre-CS baseline) in anticipation of larger reward magnitudes (Figure 1B). Analysis revealed a main effect of group, $F(2,40)=5.69, p=.007$, and a main effect of CS reward magnitude, $F(1,40)=16.27, p<.001$.

\section{Pavlovian-instrumental transfer (PIT)}

Following two sessions of instrumental retraining and one session of instrumental extinction, rats were given a PIT test, in which the 30-s CSs were presented while the 
rats were able to freely lever press without reinforcement. Figures 2A and 2B show rats' CS-induced change in lever press rate and food-port entry rate, respectively, relative to pre-CS baseline periods. Analyses revealed main effects of group, $F(2,127)=3.59, p=$ .030 , and CS reward magnitude on lever pressing, $F(1,127)=5.74, p=.018$, in which CS-induced lever pressing decreased with increases in reward magnitude. For food-port entry rate, there was a main effect of group, $F(2,127)=5.26, p=.006$, and a significant increase in CS-induced food-port entries with CS reward magnitude, $F(1,127)=8.32, p$ $=.005$.
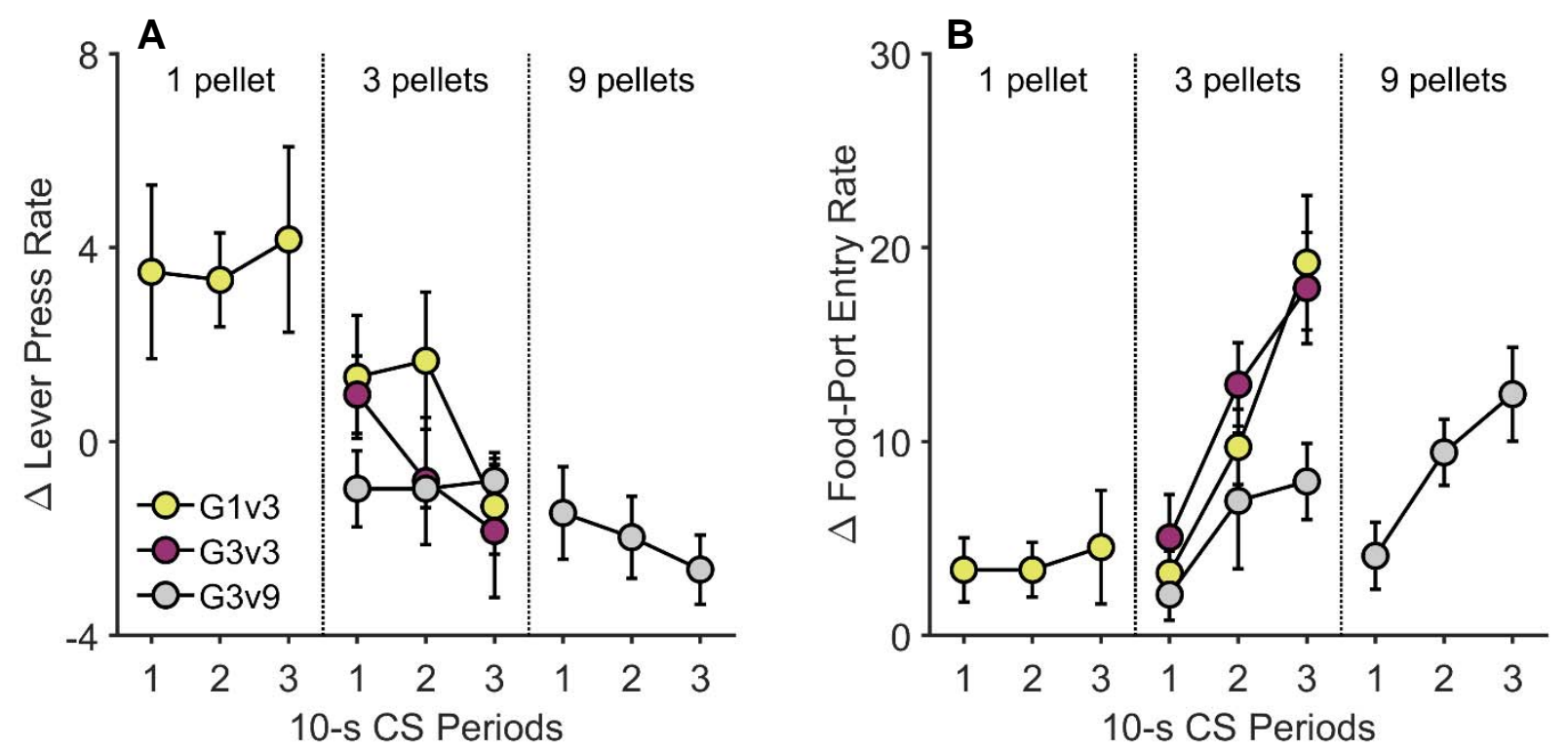

Figure 2. Pavlovian-instrumental transfer. CS-induced changes in $(A)$ lever-press rates and (B) food-port entries relative to the pre-CS baseline on the Pavlovian-instrumental transfer (PIT) test. CS $=$ conditioned stimulus. G1v3 = Group 1/3. G3v3 = Group 3/3. G3v9 = Group 3/9.

Given the between-groups manipulation of reward magnitude and that one group experienced only one reward magnitude (i.e., Group 3/3), a second set of within-group 
analyses were conducted to better elucidate how differential reward magnitude influenced CS-induced changes in behavior. For Group 1/3, the 3-pellet CS cue, relative to the 1-pellet cue, was associated with decreased lever pressing, $F(1,50)=8.37, p=$ .006 , and increased food-port entries, $F(1,50)=16.66, p<.001$, and there was also a significant CS Reward Magnitude $\times$ CS 10-s Period interaction, $F(1,50)=12.67, p=$ .001 , characterized by steeper increases in food-port entries (relative to pre-CS baseline) given the 3-pellet than 1-pellet cue. Similarly, for Group 3/9, there was decreased CS-induced lever pressing during the 9-pellet cue (compared to the 3-pellet cue), $F(1,50)=5.36, p=.025$. This was paralleled by an increased level of food-port entry during the 9- versus 3-pellet cue, $F(1,50)=4.27, p=.044$.

\section{Discussion}

The current findings shed new light on the important role that reward expectancies play in regulating the balance between instrumental reward-seeking and anticipatory rewardretrieval behaviors during the expression of PIT. We show that cues signaling a relatively small reward ( 1 pellet) elicit an immediate and sustained increase in instrumental lever pressing while producing a modest increase in food-port entry. In contrast, cues signaling either an intermediate ( 3 pellets) or large ( 9 pellets) magnitude reward tend to suppress lever pressing, particularly at the end of the cue period, near the time of expected reward delivery. Importantly, these decrements in lever pressing were associated with elevated levels of food-port entry behavior. 
These findings fit well with the view that PIT is regulated by cognitive control to ensure efficient reward retrieval based on detailed cue-evoked reward expectancies. We have previously shown that, during PIT, rats flexibly shift from pressing the lever to entering the food-port based on the expected time of reward delivery (Marshall and Ostlund, 2018), a finding replicated here. We have also shown that PIT expression critically depends on the expected probability of reward delivery, with cues signaling a low probability of reward exerting a strong motivational influence over lever pressing and cues signaling a high probability of reward preferentially eliciting food-cup approach (Marshall et al., 2020). The current findings compliment this body of work by show that expected reward magnitude also plays a role in regulating PIT.

There is good reason to use cue-related reward expectancies to regulate the organization of foraging behavior. When reward is imminent, exploratory rewardseeking behavior becomes unnecessary and should be avoided to ensure rapid retrieval of the expected reward, while it is still available. According to this view, the bias away from reward seeking and toward reward retrieval should increase when a large reward is expected, since there is more to lose in this situation. Conversely, there is less need to stop reward seeking when a small reward is expected since the potential loss is relatively low. In this case, it may be sufficient to occasionally check the food port, including after performing a bout of lever pressing.

The current findings are not readily compatible with prevailing computational models of incentive learning (Dayan and Balleine, 2002; McClure et al., 2003; Zhang et al., 2009), 
which posit that the motivational influence (or incentive salience) of a reward-predictive cue is directly linked to its state value - i.e., the total delay-discount reward expected based on that cue, as determined by temporal difference learning. While such theories make the straightforward and intuitive prediction that the conditioned motivational properties of a cue should increase with expected reward magnitude, this is at odds with the results reported here. Importantly, such theories also fail to account for previous findings that the motivational influence of reward-predictive cues on instrumental lever pressing is negatively regulated by reward expectancy in studies parametrically manipulating reward probability (Marshall et al., 2020) and timing (Marshall and Ostlund, 2018).

However, some caveats should be noted when discussing the implications of the current findings. First, we have argued that the conditioned motivational properties of reward-predictive cues are primarily reflected in their ability to facilitate performance of a separately trained instrumental reward-seeking response. While this is the central assumption of the PIT paradigm (Rescorla and Solomon, 1967; Holmes et al., 2010; Corbit and Balleine, 2016), it is possible that a cue's motivational properties also stimulate the expression of other reward-related behaviors, including attempts to retrieve reward from the food port. This would have important implications for data interpretation since both the current study and previous reports indicate that cue-elicited food-port entry rates tend to vary with expected reward value (Marshall and Ostlund, 2018; Marshall et al., 2020). However, other findings suggest that the tendency for reward-predictive cues to elicit food-port entries is not mediated by the same 
motivational process that underlies their ability to facilitate lever pressing. For instance, a growing body of PIT studies have shown that manipulations of the mesolimbic dopamine and cholinergic systems impact cue-motivated lever pressing without affecting cue-elicited food-port entry (Collins et al., 2016; Collins et al., 2019; Halbout et al., 2019). Moreover, previous studies have demonstrated that cue-elicited food-port entry behavior can be attenuated by devaluing the anticipated reward between training and testing (Holland and Rescorla, 1975; Holland, 1998; Keefer et al., 2020), indicating that its expression is mediated by a cognitive representation of the expected reward. In contrast, the excitatory influence of reward-paired cues on instrumental behavior is not sensitive to reward devaluation (Holland, 2004), in line with an implicit motivational process (e.g., general arousal).

A separate but related issue is that a cue's tendency to elicit food-port entries may interfere with its ability to motivate lever-press performance. Such response competition may undermine the ability for PIT to provide a pure readout of the motivational properties assigned to reward-predictive cues, which poses a problem for data interpretation. For instance, cues that predict large rewards may acquire greater motivational value than cues that predict a smaller rewards, but this relationship may not be apparent in PIT studies due to the presence of the competing food-port entry response. Future studies may be able to avoid this issue by using a version of the PIT task that minimizes response conflict. For instance, it has been shown that cues that signal the imminent delivery of intra-oral sucrose reward can become effective motivators of instrumental reward seeking (Lovibond, 1983), presumably because they 
do not trigger reward-retrieval activity. Similarly, extinguishing the competing food-port entry response between training and testing appears to unmask the latent motivational influence of reward-predictive cues, allowing them to invigorate reward seeking (Holmes et al., 2010). Such methods may prove useful to more accurately gauge the motivational properties of reward-predictive cues.

However, the conventional PIT task can sever as a powerful tool for parsing the unique roles that motivation and cognitive control play in cue-motivated behavior, particularly when a study is designed to vary the degree of conflict between the lever-press and food-port entry responses across cue conditions. We have previously used cue-specific variations in reward probability (Marshall et al., 2020) and reward timing (Marshall and Ostlund, 2018) to probe developmental and drug-induced aberrations in cue-motivated behavior. The current study indicates that cue-specific variations in reward magnitude may also prove useful for such studies. 


\section{References}

Azrin NH, Hake DF (1969) Positive Conditioned Suppresion - Conditioned Suppression Using Positive Reinforcers as Unconditioned Stimuli. J Exp Anal Behav 12:167173.

Boisgontier MP, Cheval B (2016) The anova to mixed model transition. Neurosci Biobehav Rev 68:1004-1005.

Bolker BM, Brooks ME, Clark CJ, Geange SW, Poulsen JR, Stevens MHH, White J-SS (2009) Generalized linear mixed models: a practical guide for ecology and evolution. Trends in Ecology \& Evolution 24:127-135.

Cartoni E, Balleine B, Baldassarre G (2016) Appetitive Pavlovian-instrumental Transfer: A review. Neurosci Biobehav Rev 71:829-848.

Collins AL, Aitken TJ, Greenfield VY, Ostlund SB, Wassum KM (2016) Nucleus Accumbens Acetylcholine Receptors Modulate Dopamine and Motivation. Neuropsychopharmacology 41:2830-2838.

Collins AL, Aitken TJ, Huang IW, Shieh C, Greenfield VY, Monbouquette HG, Ostlund SB, Wassum KM (2019) Nucleus Accumbens Cholinergic Interneurons Oppose Cue-Motivated Behavior. Biol Psychiatry 86:388-396.

Colwill RM, Rescorla RA (1988) Associations between the discriminative stimulus and the reinforcer in instrumental learning. Journal of Experimental Psychology: Animal Behavior Processes 14:155-164.

Corbit LH, Janak PH (2007) Ethanol-associated cues produce general pavlovianinstrumental transfer. Alcohol Clin Exp Res 31:766-774.

Corbit LH, Balleine BW (2016) Learning and Motivational Processes Contributing to Pavlovian-Instrumental Transfer and Their Neural Bases: Dopamine and Beyond. Curr Top Behav Neurosci 27:259-289.

Corbit LH, Janak PH (2016) Changes in the Influence of Alcohol-Paired Stimuli on Alcohol Seeking across Extended Training. Front Psychiatry 7:169.

Crombag HS, Galarce EM, Holland PC (2008) Pavlovian influences on goal-directed behavior in mice: The role of cue-reinforcer relations. Learn Memory 15:299-303. Dayan P, Balleine BW (2002) Reward, motivation, and reinforcement learning. Neuron 36:285-298. 
Garbusow M et al. (2016) Pavlovian-to-instrumental transfer effects in the nucleus accumbens relate to relapse in alcohol dependence. Addiction Biology 21:719731.

Genauck A, Andrejevic M, Brehm K, Matthis C, Heinz A, Weinreich A, Kathmann N, Romanczuk-Seiferth N (2020) Cue-induced effects on decision-making distinguish subjects with gambling disorder from healthy controls. Addict Biol 25:e12841.

Halbout B, Marshall AT, Azimi A, Liljeholm M, Mahler SV, Wassum KM, Ostlund SB (2019) Mesolimbic dopamine projections mediate cue-motivated reward seeking but not reward retrieval in rats. Elife 8:e43551.

Hoffman L, Rovine MJ (2007) Multilevel models for the experimental psychologist:

Foundations and illustrative examples. Behavior Research Methods 39:101-117.

Holland P (1998) Amount of training affects associatively-activated event representation. Neuropharmacology 37:461-469.

Holland PC (2004) Relations between Pavlovian-instrumental transfer and reinforcer devaluation. J Exp Psychol Anim Behav Process 30:104-117.

Holland PC, Rescorla RA (1975) The effect of two ways of devaluing the unconditioned stimulus after first- and second-order appetitive conditioning. Journal of Experimental Psychology: Animal Behavior Processes 1:355-363.

Holmes NM, Marchand AR, Coutureau E (2010) Pavlovian to instrumental transfer: a neurobehavioural perspective. Neurosci Biobehav Rev 34:1277-1295.

Keefer SE, Bacharach SZ, Kochli DE, Chabot JM, Calu DJ (2020) Effects of Limited and Extended Pavlovian Training on Devaluation Sensitivity of Sign- and GoalTracking Rats. Frontiers in Behavioral Neuroscience 14:3.

LeBlanc KH, Ostlund SB, Maidment NT (2012) Pavlovian-to-instrumental transfer in cocaine seeking rats. Behav Neurosci 126:681-689.

LeBlanc KH, Maidment NT, Ostlund SB (2013) Repeated cocaine exposure facilitates the expression of incentive motivation and induces habitual control in rats. PLoS One 8:e61355. 
LeBlanc KH, Maidment NT, Ostlund SB (2014) Impact of repeated intravenous cocaine administration on incentive motivation depends on mode of drug delivery. Addict Biol 19:965-971.

Lovibond PF (1981) Appetitive Pavlovian-Instrumental Interactions - Effects of InterStimulus Interval and Baseline Reinforcement Conditions. Q J Exp Psychol-B 33:257-269.

Lovibond PF (1983) Facilitation of instrumental behavior by a Pavlovian appetitive conditioned stimulus. J Exp Psychol Anim Behav Process 9:225-247.

Marshall AT, Ostlund SB (2018) Repeated cocaine exposure dysregulates cognitive control over cue-evoked reward-seeking behavior during Pavlovian-toinstrumental transfer. Learn Mem 25:399-409.

Marshall AT, Munson CN, Maidment NT, Ostlund SB (2020) Reward-predictive cues elicit excessive reward seeking in adolescent rats. Dev Cogn Neurosci 45:100838.

McClure SM, Daw ND, Montague PR (2003) A computational substrate for incentive salience. Trends Neurosci 26:423-428.

Ostlund SB, LeBlanc KH, Kosheleff AR, Wassum KM, Maidment NT (2014) Phasic mesolimbic dopamine signaling encodes the facilitation of incentive motivation produced by repeated cocaine exposure. Neuropsychopharmacology 39:24412449.

Pinheiro JC, Bates DM (2000) Mixed-effects models in S and S-PLUS. New York: Springer.

Rescorla RA (1994) Transfer of instrumental control mediated by a devalued outcome. Animal Learning \& Behavior 22:27-33.

Rescorla RA, Solomon RL (1967) Two-process learning theory: Relationships between Pavlovian conditioning and instrumental learning. Psychol Rev 74:151-182.

Saddoris MP, Stamatakis A, Carelli RM (2011) Neural correlates of Pavlovian-toinstrumental transfer in the nucleus accumbens shell are selectively potentiated following cocaine self-administration. European Journal of Neuroscience 33:2274-2287. 
Schielzeth H, Nakagawa S, Freckleton R (2013) Nested by design: model fitting and interpretation in a mixed model era. Methods in Ecology and Evolution 4:14-24.

Sebold M, Garbusow M, Cerci D, Chen K, Sommer C, Huys QJ, Nebe S, Rapp M, Veer IM, Zimmermann US, Smolka MN, Walter H, Heinz A, Friedel E (2021) Association of the OPRM1 A118G polymorphism and Pavlovian-to-instrumental transfer: Clinical relevance for alcohol dependence. J Psychopharmacol:269881121991992.

Shiflett MW (2012) The effects of amphetamine exposure on outcome-selective Pavlovian-instrumental transfer in rats. Psychopharmacology (Berl) 223:361-370. Shiflett MW, Riccie M, DiMatteo R (2013) The effects of amphetamine sensitization on conditioned inhibition during a Pavlovian-instrumental transfer task in rats. Psychopharmacology (Berl) 230:137-147.

Tatham TA, Zurn KR (1989) The MED-PC experimental apparatus programming system. Behavior Research Methods, Instruments, \& Computers 21:294-302.

Vandyne GC (1971) Conditioned Suppression with a Positive Us in Rat. J Comp Physiol Psych 77:131-\&.

Vogel V, Kollei I, Duka T, Snagowski J, Brand M, Muller A, Loeber S (2018) Pavlovianto-instrumental transfer: A new paradigm to assess pathological mechanisms with regard to the use of Internet applications. Behav Brain Res 347:8-16. Wyvell CL, Berridge KC (2001) Incentive sensitization by previous amphetamine exposure: increased cue-triggered "wanting" for sucrose reward. J Neurosci 21:7831-7840.

Zhang J, Berridge KC, Tindell AJ, Smith KS, Aldridge JW (2009) A neural computational model of incentive salience. PLoS Comput Biol 5:e1000437. 